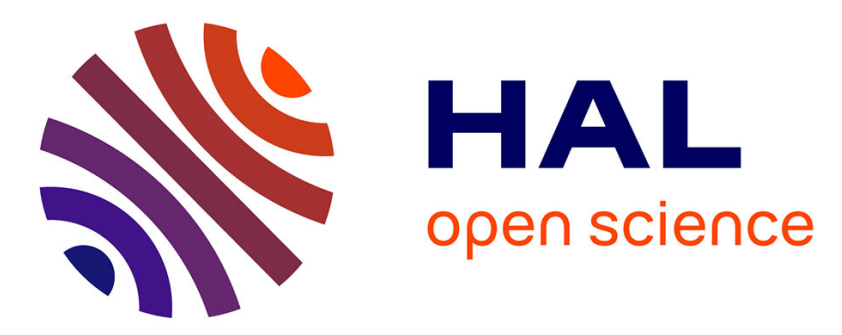

\title{
Failure time, critical behaviour and activation processes in crack formation
}

\author{
Sergio Ciliberto, Stéphanie Deschanel, Alessio Guarino, Stéphane Santucci, \\ Riccardo Scorretti, Loïc Vanel
}

\section{> To cite this version:}

Sergio Ciliberto, Stéphanie Deschanel, Alessio Guarino, Stéphane Santucci, Riccardo Scorretti, et al. Failure time, critical behaviour and activation processes in crack formation. 11th International Conference on Fracture, Mar 2005, Turin, Italy. ensl-00179855

\section{HAL Id: ensl-00179855}

\section{https://hal-ens-lyon.archives-ouvertes.fr/ensl-00179855}

Submitted on 16 Oct 2007

HAL is a multi-disciplinary open access archive for the deposit and dissemination of scientific research documents, whether they are published or not. The documents may come from teaching and research institutions in France or abroad, or from public or private research centers.
L'archive ouverte pluridisciplinaire HAL, est destinée au dépôt et à la diffusion de documents scientifiques de niveau recherche, publiés ou non, émanant des établissements d'enseignement et de recherche français ou étrangers, des laboratoires publics ou privés. 


\title{
Failure time, critical behaviour and activation processes in crack formation
}

\author{
S. Ciliberto ${ }^{1 *}$ S. Deschanel ${ }^{2}$, A.Guarino ${ }^{3}$, \\ S. Santucci ${ }^{1}$, R. Scorretti ${ }^{4}$, L. Vanel ${ }^{1}$ \\ 1 Laboratoire de Physique, CNRS UMR5672, Ecole Normale Supérieure de Lyon, \\ 46 allée d'Italie, 69364 Lyon, France \\ 2 GEMPPM, INSA de Lyon, 69621 Villeurbanne, France \\ 3 Université de Polynésie Française - BP 6570 FAA'A, Tahiti, French Polynesia \\ ${ }^{4}$ Ecole Centrale de Lyon, 69134 Ecully, France
}

In this seminar we will review several experimental and theoretical aspects of two very important problems of material failure: crack precursors and crack prediction. We will summarize the results of several experiments and numerical simulations that we have performed in order to give new insight on these two important problems. Specifically the acoustic emission of fracture precursors, and the failure time of samples made of heterogeneous materials (wood, fiberglass) are studied as a function of the load features and geometry[1, 2, 3, 4]. The accurate study of the localization and of the statistics of acoustic emission is indeed very important in order to compare the experimental results with those of percolation and critical models for crack formation. A typical example of microcrack localization in a large cylindrical sample is described in fig.1 Once the microcracks have been localized we study the statistics of the time interval $\delta t$ between events (precursors) and the acoustic energy $\varepsilon$ of each event. We find that they are power law distributed and that the exponents of these power laws depend on the load history and on the material. An example is shown in fig.2 $[1,2,3,4]$

\footnotetext{
${ }^{*}$ Corresponding author: sergio.ciliberto@enslyon.fr
}

When the sample failure is produced by an imposed stress (see fig.3a) the cumulative acoustic energy $E$, that is the integral of $\varepsilon$ as a function of time, presents a critical divergence near the failure time $\tau$, which is

$$
E \sim\left(\frac{\tau-t}{\tau}\right)^{-\gamma} .
$$

It is important to point out that this critical behavior is observed only if the stress is imposed, whereas no power law is observed when the strain is imposed,see fig.3b). Notably the positive exponent $\gamma$, in eq.1, is independent, within error bars, on all the experimental parameters (loading speed and materials). The failure time is, of course a function of the applied stress, which can be constant or a function of time. If a constant pressure $\mathrm{P}$ is applied to a sample, we found that in heterogeneous materials the failure time $\tau$ has a functional dependence on $\mathrm{P}$ which is predicted with a good accuracy by a model of microcrack nucleation proposed by Pomeau $[3,4]$, specifically:

$$
\tau=\tau_{o} \exp \left(\frac{P_{o}}{P}\right)^{4}
$$

where $P$ is the external constant pressure applied to the sample and 


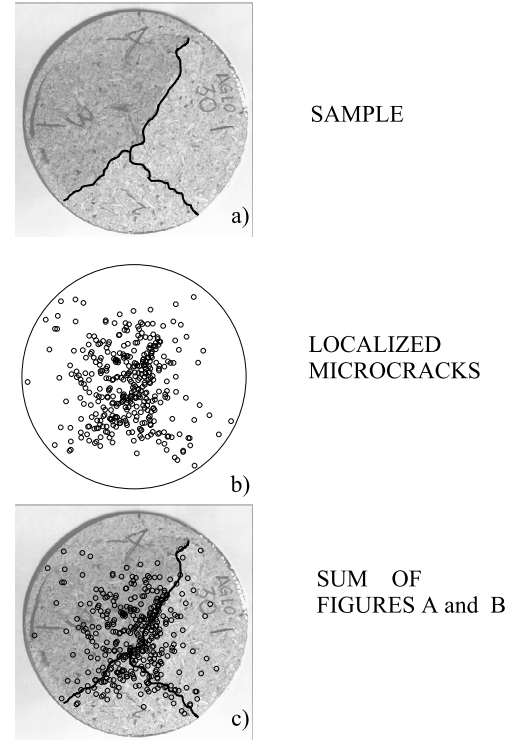

Figure 1: Microcrack localization Example of localization in a cylindrical sample of diameter $20 \mathrm{~cm}$ and thickness $0.3 \mathrm{~cm}$. The samples are broken by a stress perpendicular to the base of the cylinder $[1,2,3,4]$. In a) the broken wood sample is shown. We clearly distinguish the main final cracks. When a sample is submitted to an external stress, sound is emitted by microcracks appearing before the final failure. In b) the small circles represent the sources of acoustic waves detected and localized by mean of four detectors. In c), we superpose the figure a) and b). We clearly see that the density of acoustic sources (microcracks) is very large on the final cracks. The final crack is produced by the nucleation of many microcracks. This picture is qualitatively similar to that of percolation models for cracks.
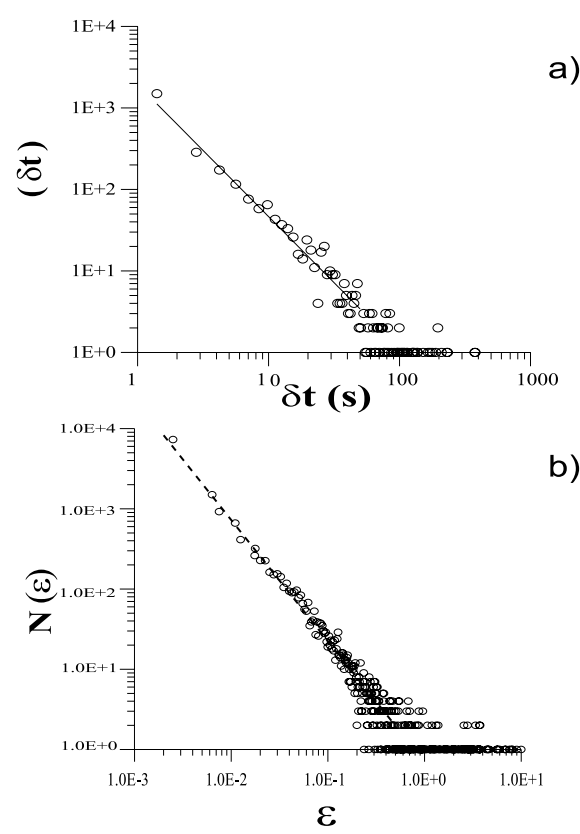

Figure 2: Statistics of the acoustic emissions of microcracks. a) Distribution of the time $\delta t$ between two acoustic emissions occurring during the slow crack formation.b) distribution of the acoustic energy $\varepsilon$ emitted by each microcrack 


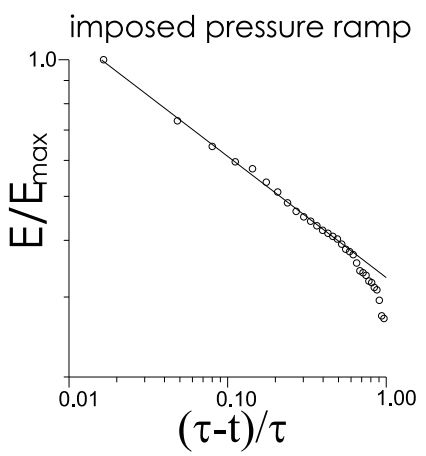

imposed strain ramp

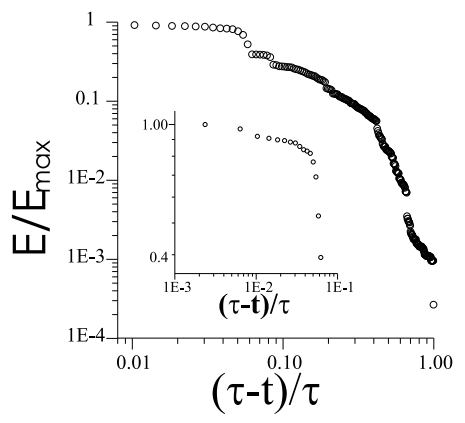

Figure 3: Cumulative acoustic energy of microcracks. The cumulative acoustic energy is plotted as a function of the reduced time $\left(\frac{\tau-t}{\tau}\right)$, where $\tau$ is the failure time of the sample. a) The sample is submitted to a linearly increasing pressure ramp. b) The sample is submitted to a linearly increasing strain ramp. We clearly see that in a) $E \sim\left(\frac{\tau-t}{\tau}\right)^{-\gamma}$. The exponent $\gamma$ is independent within error bars by the loading speed and by the sample material. No critical behaviour is observed in b) when the strain is imposed

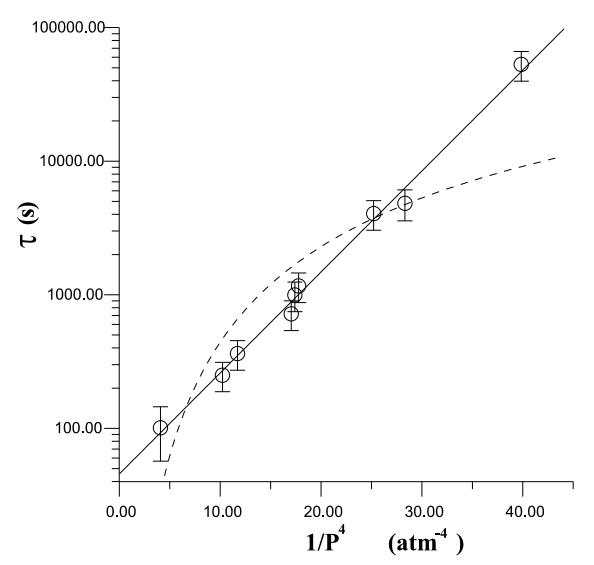

Figure 4: Life time of a sample submitted at a constant external pressure $P$. The life time $\tau$ is plotted as a function of $P^{-4}$. The continuous line is the fit with eq.2. The dashed line is a best fit of the data using Mori or Zurkov laws. We clearly see that the fit with eq. 2 is much better than the other ones

$P_{o}^{4} \simeq \Gamma^{3} Y^{2} /\left(K_{B} T\right)$ is a parameter which depends on the temperature T, the Young modulus $\mathrm{Y}$ and the surface energy $\Gamma$ of the material. A typical result of the experiments is shown in fig.4, where the life time of a large cylindrical sample is plotted as a function of $P^{-4}$. We see the excellent agreement between the experimental points and the fit performed using eq. 2 . We have extended this model to time dependent $P$ (see ref. $[3,4]$ for details) and we find an excellent agreement with the experimental data. It is interesting to notice that even in the case when a constant pressure is applied to the sample the cumulative acoustic energy $\mathrm{E}$ has the dependence on time described by eq.1. Thus the dependence of $E$ on the reduced time $(\tau-t) / \tau$, shown in fig. 3 , turns out to be a new type of critical behavior where the control parameter is only the time [4]. All the other parameter of the experiments are indeed taken into account by $\tau$. In spite of the good agreement between the experimental data of $\tau$ and the functional dependence 
on $P$, given by eq.2, the activation model fails to reproduce in a quantitative way the experimental results, mainly in the temperature dependence [3, 4]. Motivated by this discrepancy we have investigated in simple models (the fiber bundle model) the role of disorder on the fracture produced by a thermal activation of microcracks $[5,6]$. We find that the presence of disorder (heterogeneity) contributes to increasing the effective temperature of a sample subject to a constant load. Equivalently, but perhaps more physically, one can state that disorder renormalizes the barrier height to be overcome in order to give rise to a macroscopic failure of the fiber bundle. This scenario can be understood by noticing that the fracture evolves through a sequence of many irreversible processes. After the failure of the weakest fibers, the system cannot any longer come back to its initial state, while, at the same time, the energy barrier has lowered. Although the fiber models explain the existence of a large effective temperature, they present a dependence on $P$ which is quite different from that of eq.2, which is not yet completely understood.

In order to give new insight into this problem we have studied, the role of an activation processes in the propagation of a single crack in a thin sheet of papers submitted to a tensile force $F$. The statistical and mean dynamical properties turn out to be in excellent agreement with the numerical and analytical results of a $2 \mathrm{D}$ elastic model where crack growth is controlled by the thermal noise $[7,8]$. In the experiment, we observe that the crack progresses by jumps as it can be seen in fig. $5 \mathrm{a}$ ), where time is plotted as a function of the crack length $L$. The mean behaviour, obtained as the average of many experiments performed in the same conditions, is plotted in the insert of fig.5a). The dotted line is the prediction of the $2 \mathrm{D}$ elastic model [7], that is

$$
t=\tau\left[1-\exp \left(\frac{L_{i}-L}{l_{o}}\right)\right]
$$

where $L_{i}$ is the initial crack length, $l_{o}$ a characteristic length, and $\tau$ is the life time of the sample. The experimental data plotted in the insert of fig.5a) are in very good agreement with the functional form of eq.3. The $2 \mathrm{D}$ elastic model of crack thermal activation predicts also the probability density function $p(s)$ of the crack jump sizes $s$. An example is given in fig. $5 \mathrm{~b}$ ), where $p(s)$ are plotted for various values of the stress intensity factor $K=F /(W h) \sqrt{(} L) g(L / W)$. Here $W$ is the width of the sample, $h$ its thickness and $g(L / W)$ takes into account the finite size effects. We see the good agreement between the measured PDF and the predicted ones. Finally the 2D model predicts for $\tau$ the following dependence on $K_{i}$ ( $K$ at $L=L_{i}$ ).

$$
\tau=\tau_{o} \exp \left[-\frac{\alpha\left(K_{c}-K_{i}\right)^{2}}{2 Y K_{B} T}\right]
$$

where $K_{c}$ is the toughness of the material, that is its critical stress intensity factor. The measured $\tau$ of thin sheets of paper seem to have the functional dependence on $K_{i}$ of eq.4, but at the moment the statistic on $\tau$ is not yet enough to make an accurate statement.

As a conclusion, we have shown that slow crack nucleation in heterogeneous materials presents, close to the failure time of the samples, several critical features. We have also seen that the real control parameter of this critical behavior is time and all the other parameters of the experiments (elastic properties of the materials, loading speed etc) are taken into account by the time of life $\tau$. The functional behaviour of $\tau$ on the applied stress is predicted by activation models, which, in the case of experiments in thin sheets of papers, can predict also the statistics of crack jumps and the mean behaviour of the crack. However many problems are still open for understanding the role of activation processes in crack formation. The main one is the dependence on temperature of the life time. Indeed in all of the experiments the temperature estimated by the models is much higher than the real one. We 
a)

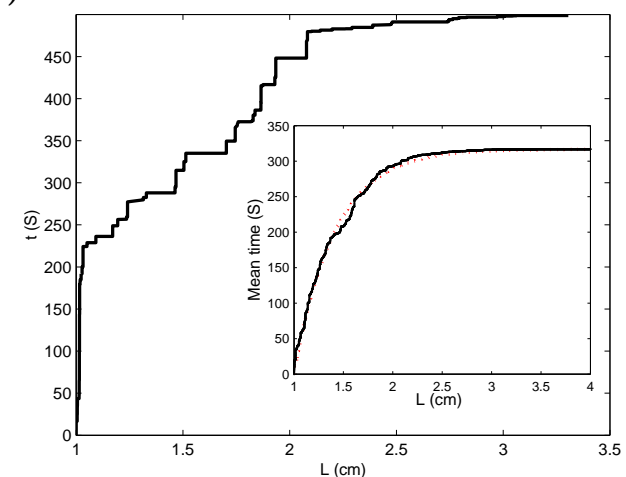

b)

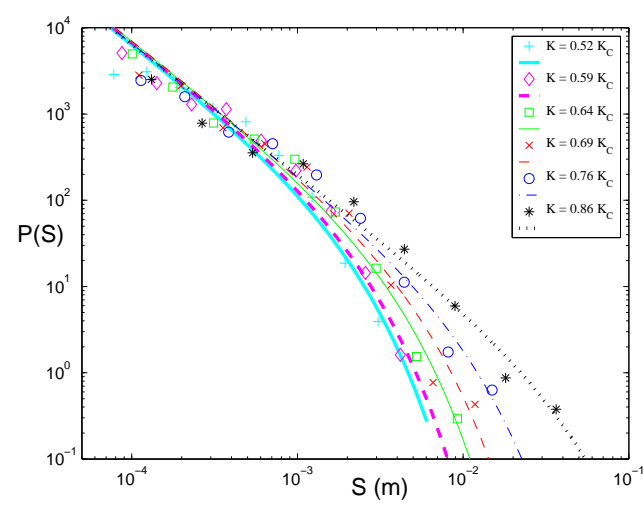

Figure 5: Experiment on the slow crack propagation on a sheet of paper. a) Time is plotted as function of the crack length for a single experiment. The plot shows crack jumps and crack arrest. In insert, average time to reach L (10 experiments with $F=270 N$ and $L_{i}=1 \mathrm{~cm}$ ) and prediction for thermally activated growth from eq.3 [7] (dotted curve). b) Step size distributions (symbols) for various values of the stress intensity factor. The continuous lines are best fits of experimental data done using the predictions of the $2 \mathrm{D}$ elastic model of thermally activated crack. have shown that, in simple models, the disorder of the material strongly enhances the role of thermal fluctuations. However the correspondence of these theoretical results on real systems is still unclear. Furthermore there is another important open problem, specifically the different dependence of $\tau$ on the applied stress for systems close to 2D (eq.4) and for $3 \mathrm{D}$ systems (eq.2). Both equations seem to fit the experimental data. However one may wonder whether eq.2 is an approximation of a much more complex behaviour. The final question concerns the role of viscoelasticity in the delayed crack. This role is still unclear in these systems. All these problems requires many new experiments in order to solve them. Work is in progress.

\section{References}

[1] Statistical analysis of crack precursors, A. Garcimartin, L. Bellon, A. Guarino, S. Ciliberto, Phys. Rev. Lett., 79, 3202 (1997).

[2] An Experimental Test of the Critical Behaviour of Fracture Precursors, A. Guarino, A. Garcimartin, S. Ciliberto, Eur. Phys. J. B 6, 13 (1998). .

[3] Failure time and microcrack nucleation, A. Guarino, A. Garcimartin, S. Ciliberto, Europhysics Letters, 47, 456 (1999).

[4] Failure time and critical behaviour of fracture precursors in heterogeneous materials, A. Guarino, S. Ciliberto, A. Garcimartin, M. Zei, R. Scorretti, European Physical Journal, B 26, 141-151 (2002)

[5] The effect of disorder on the fracture nucleation process, A. Guarino, R. Scorretti, S. Ciliberto, Physica D 158, 83-104 (2001).

[6] Failure time in the fiber-bundle model with thermal noise and disor- 
der,A.Politi, S. Ciliberto,R. Scorretti, Phys. Rev. E. 66 (2), 026107, (2002)

[7] Thermal activation of rupture and slow crack growth in a model of homogeneous brittle materials, S. Santucci, L. Vanel, A. Guarino, R. Scorretti, S. Ciliberto, Europhysics Letters Vol.62, 3, 320 (2003)

[8] Sub-critical statistics in rupture of fibrous materials : model and experiments. S. Santucci, L. Vanel, S. Ciliberto, submitted. 